43-44 | 2013

Le pastoralisme en Haute-Asie : la raison nomade dans l'étau des modernisations

\title{
Powers John and Templeman David, Historical Dictionary of Tibet
}

The Scarecrow Press, Inc. Lanham-Toronto-Plymouth, UK., 2012. ISBN

9780810868052

Katia Buffetrille

\section{OpenEdition}

Édition électronique

URL : https://journals.openedition.org/emscat/2189

DOI : $10.4000 /$ emscat.2189

ISSN : 2101-0013

Éditeur

Centre d'Etudes Mongoles \& Sibériennes / École Pratique des Hautes Études

Référence électronique

Katia Buffetrille, «Powers John and Templeman David, Historical Dictionary of Tibet », Études mongoles et sibériennes, centrasiatiques et tibétaines [En ligne], 43-44 | 2013, mis en ligne le 20 septembre 2013, consulté le 13 juillet 2021. URL : http://journals.openedition.org/emscat/2189; DOI : https://doi.org/ 10.4000/emscat.2189

Ce document a été généré automatiquement le 13 juillet 2021.

(c) Tous droits réservés 


\section{Powers John and Templeman David, Historical Dictionary of Tibet}

The Scarecrow Press, Inc. Lanham-Toronto-Plymouth, UK., 2012. ISBN

9780810868052

Katia Buffetrille

\section{RÉFÉRENCE}

Powers John and Templeman David, Historical Dictionary of Tibet, The Scarecrow Press, Inc. Lanham-Toronto-Plymouth, UK., 2012

1 C'est un véritable défi qu'ont relevé J. Powers et D. Templeman en composant ce Dictionnaire historique du Tibet qui couvre, en réalité, un champ beaucoup plus vaste que celui annoncé dans le titre.

2 Ce dictionnaire appartient à une série qui traite, à ce jour, de l'Asie, de l'Océanie et du Moyen-Orient. Un volume dédié au Tibet s'imposait, et l'on ne peut que remercier les deux auteurs d'avoir entrepris cet énorme travail. Comme il est d'usage dans la collection, la couverture représente le drapeau du pays concerné, qui porte ici l'emblème créé au temps du xiII dalai lama (1876-1933).

Les auteurs offrent une chronologie (pp. XXIX-XXXVII) qui souligne les événements les plus marquants de l'histoire tibétaine. Profitant des récentes découvertes archéologiques, ils la font débuter à l'époque néolithique et la poursuivent jusqu'en 2011. En ce qui concerne la période récente, les auto-immolations de Tibétains par le feu qui ont marqué les années 1998, 2007, 2009 et 2011 (date à laquelle s'arrête cette chronologie) ne sont malheureusement pas mentionnées. Elles marquent pourtant un tournant dans l'histoire de la lutte des Tibétains contre le gouvernement chinois, au Tibet comme en exil.

4 Une introduction (pp. 1-45) permet au lecteur de se familiariser avec la géographie, les mythes, et surtout l'histoire complexe de ce pays qu'est le Tibet. N'eût-il pas été nécessaire de distinguer plus clairement pour le non-spécialiste les faits qui relèvent de 
la tradition tibétaine et ceux qui se rapportent à l'histoire réelle ? Par exemple, la date de 233 est donnée comme la date réelle à laquelle textes bouddhiques et reliques seraient tombés sur le palais du roi mythique Lha tho tho ri (p. XXIX), alors que dans le paragraphe qui lui est consacré (p. 405), les auteurs précisent bien que c'est l'histoire selon la tradition tibétaine. De même, il aurait fallu préciser que l'histoire de Padmasambhava et de ses voyages telle qu'elle est évoquée dans l'introduction appartient à la légende (p. 20).

都 connaissances; elle couvre divers domaines: histoire, anthropologie, architecture, archéologie, art, langage, littérature et religion.

La plus grande part du dictionnaire est composée de notices consacrées aux personnages importants de l'histoire tibétaine sur lesquels sont données les informations essentielles. Rappelons qu'à ce jour, aucun ouvrage de ce type n'existait en langue occidentale : les deux auteurs ont donc non seulement comblé un grand vide, mais répondu à une attente. Ils ont, par ailleurs, dépassé le cadre strictement historique et intégré à leur dictionnaire les noms des grandes divinités du bouddhisme tibétain, ce qui se justifie pour un pays comme le Tibet, dans lequel la religion a façonné la civilisation et pénétré la plupart des activités de la vie quotidienne. Une note eût cependant été la bienvenue pour expliquer la démarche extensive des auteurs et les raisons qui les ont poussés à étendre ce dictionnaire historique à d'autres champs de la culture tibétaine.

7 Il est probable que le choix de mettre les entrées en lettres capitales vient d'un souci d'harmonisation avec les autres volumes. Cela pose de véritables problèmes en tibétain, car l'écriture étant très complexe du fait de l'ajout de préfixes, suffixes, lettres souscrites et suscrites qui ne se prononcent pas, le lecteur non-tibétologue sera vite découragé devant ces séries de termes qui paraissent totalement imprononçables. Il est probable que si, seule la première lettre qui se prononce, avait été notée en majuscule et les autres en minuscules, le lecteur non spécialiste aurait été moins perdu. On peut déplorer d'ailleurs le recours, pour les entrées des termes répertoriés, à la translittération scientifique au lieu d'une transcription simplifiée, plus phonétique, qui aurait mieux convenu à ce type d'ouvrage. C'est, en effet, un obstacle supplémentaire à l'utilisation du dictionnaire par un non-tibétologue, et cela d'autant plus que les termes sont classés selon l'ordre de l'alphabet occidental. Les auteurs ont sans doute été conscients de cette difficulté, puisqu'ils ont inclus (pp. XVII-XX) une liste qui permet d'établir la correspondance entre transcription phonétique et translittération des termes tibétains répertoriés. D'une certaine manière, il fait aussi office d'index des personnages, lieux et divinités auxquels les auteurs de ce dictionnaire ont consacré des entrées.

On peut enfin vivement regretter que dans un dictionnaire consacré au Tibet, et qui se veut donc un outil pour améliorer la connaissance que l'on a de ce pays, la graphie tibétaine n'ait pas été donnée (alors que les caractères usités pour les transcriptions en chinois sont, eux, bien indiqués).

Comme dans toute entreprise de cette ampleur, le volume présente quelques coquilles : une relecture attentive aurait, par exemple, permis d'éviter de citer Sir Marc Aurel Stein comme auteur d'une étude sur Gesar de Gling, au lieu de R.A Stein.

Dans le cas d'une réimpression, il serait bon de reconsidérer le public auquel est destiné ce dictionnaire. En indiquant les entrées en translittération scientifique, les auteurs 
ferment la porte à un large public de non-spécialistes et de curieux qui aurait certainement pris beaucoup d'intérêt à ce livre. Dans cette perspective, l'ajout d'un véritable index ne nuirait pas afin d'aider le lecteur dans sa recherche.

11 Ces quelques remarques ne sauraient diminuer la valeur et la richesse de ce premier dictionnaire historique du Tibet, qui devrait se trouver dans toute bibliothèque sur le Pays des Neiges. 